\title{
THE WEIERSTRASS THEOREM IN FIELDS WITH VALUATIONS
}

\author{
IRVING KAPLANSKY 1
}

In $[2 \text {, Theorem 32 }]^{2}$ the author showed that an analogue of the Weierstrass-Stone theorem holds in topological rings having ideal neighborhoods of 0 . Earlier, Dieudonné [1] had proved the Weierstrass-Stone theorem for the field of $p$-adic numbers. Now the field of $p$-adic numbers has an open subring (the $p$-adic integers) with ideal neighborhoods of 0 . It seems plausible, therefore, to expect that the method of [2] will apply, provided one has a supplementary device for "getting into" the $p$-adic integers. This is in fact the case, and the result is applicable to any division ring with a valuation of rank one. ${ }^{2}$ The requisite lemma reads as follows:

LEMMA 1. Let $F$ be a division ring with a valuation of rank one, and $B$ its valuation ring. Let $a$ be any nonzero element in $F$, and $K$ a compact subset of $F$. Then there exists a (non-commutative) polynomial $f$ with coefficients in $F$, without a constant term, and satisfying $f(a)=1$, $f(K) \subset B$.

Proof. Let $P$ denote the maximal ideal in $B$, that is, the set of all elements $x$ with $V(x)>0$, where $V$ denotes the valuation. Let $K^{\prime}$ denote the subset of $K$ with values less than $V(a) ; K^{\prime}$ will again be compact. For any $c$ in $K^{\prime}$ there is a compact open subset of $K^{\prime}$ containing $c$ and contained in $c(1+P)$. Take a finite covering of $K^{\prime}$ consisting of such neighborhoods: say $U_{1}, \cdots, U_{r}$ with $U_{i}$ contained in $c_{i}(1+P)$. Suppose the $c$ 's numbered so that $V\left(c_{i}\right) \geqq V\left(c_{i+1}\right)$. Now $1-c_{i}^{-1} U_{i}$ is a compact subset of $P$, and consequently the values of its elements have a positive lower bound $\alpha_{i}$. Choose integers $n(1), \cdots, n(r)$ in succession large enough so that

$$
V\left(a^{-1} c_{i}\right)+\sum_{j=1}^{i-1} n(j) V\left(c_{j}^{-1} c_{i}\right)+n(i) \alpha_{i} \geqq 0
$$

for $i=1, \cdots, r$. Then the polynomial

$$
f(x)=1-\left(1-a^{-1} x\right)\left(1-c_{1}^{-1} x\right)^{n(1)} \cdots\left(1-c_{r}^{-1} x\right)^{n(r)}
$$

satisfies the requirements of the lemma.

Received by the editors April 18, 1949.

1 John Simon Guggenheim Memorial Fellow.

2 Numbers in brackets refer to the bibliography at the end of the paper.

That is to say, a valuation whose value group is a subgroup of the real numbers. 
We shall restate [2, Theorem 32 ] in a slightly sharpened form.

Lemma 2. Let $A$ be a topological ring with unit element and ideal neighborhoods of 0 , let $X$ be a totally disconnected locally compact Hausdorff space, and $C$ the ring of all continuous functions from $X$ to $A$ vanishing at $\infty$. Topologize $C$ by uniform convergence. Let $D$ be a closed subring of $C$, containing for any distinct points $x, y \in X$ and any $a, b$ $\in A$, a function $f$ with $f(x)=a, f(y)=b$. Then $D=C$.

Proor. Let $U$ be a fixed ideal neighborhood of $0,1 \notin U+U$. Let $K$ be a compact subset of $X$ and $x$ a point not in $K$. Then $D$ contains a function $f$ with $f(x)=1$ and $f(y)=0$ for a given $y$ in $K$. The function $f$ will take values in $U$ in a suitable neighborhood of $y$. A finite number of these neighborhoods cover $K$; if $g$ is the product of the corresponding $f$ 's, we have $g(x)=1, g(K) \subset U$.

We start again with an arbitrary $z \in X$ and an $h$ in $D$ with $h(z)=1$. There is a compact neighborhood $L$ of $z$ with $h(L) C 1+U$, and a larger compact set $M$ such that $h$ is in $U$ in the complement of $M$. For any given $w$ in $M-L$ we can, by the preceding paragraph, find a function $p$ in $D$ with $p(L) \subset U, p(w)=h(w)$. Then the function $h(h-p)$ has the following properties: in $L$ its values lie in $1+U$, and it vanishes at $w$ and accordingly lies in $U$ in a neighborhood of $w$. A finite number of these neighborhoods cover the compact set $M-L$. The product of the corresponding elements $h(h-p)$ gives us an element $q$ in $D$ with $q(L) \subset 1+U, q\left(L^{\prime}\right) \subset U, L^{\prime}$ the complement of $L$. By combining such elements we can get "within $U^{\text {n }}$ of the characteristic function of any compact open set. Since $D$ is closed, it actually contains all characteristic functions of compact open sets, from which it follows readily that $D=C$.

As an immediate consequence of Lemmas 1 and 2, we have the following generalization of Dieudonné's theorem:

TheOREM. Let $F$ be a division ring with a valuation of rank one, $X$ a totally disconnected locally compact Hausdorff space, $C$ the ring of all continuous functions from $X$ to $F$ vanishing at $\infty$. Topologize $C$ by uniform convergence. Let $D$ be a closed subring of $C$, admitting left-multiplication by the constant functions, and containing for any two distinct points $x, y \in X$ a function vanishing at $x$ but not at $y$. Then $D=C$.

\section{Biblography}

1. J. Dieudonne, Sur les fonctions continues p-adiques, Bull. Sci. Math. (2) vol. 68 (1944) pp. 79-95.

2. I. Kaplansky, Topological rings, Amer. J. Math. vol. 69 (1947) pp. 153-183.

INSTITUTE FOR AdVANCED STUdY 